\title{
Albumin-to-bilirubin score for assessing the in-hospital death in cirrhosis
}

\author{
Lichun Shao ${ }^{*}$, Bing $\mathrm{Han}^{1 *}$, Shu $\mathrm{An}^{2}$, Jiaxin $\mathrm{Ma}^{1}$, Xiaozhong Guo ${ }^{3}$, Fernando Gomes Romeiro ${ }^{4}$, Andrea \\ Mancuso ${ }^{5,6}$, Xingshun $\mathrm{Qi}^{3}$ \\ ${ }^{1}$ Department of Gastroenterology, No. 463 Hospital of Chinese PLA, Shenyang 110000, China; ${ }^{2}$ Medical Office, Xingcheng Sanitarium of Shenyang \\ Military Area, Xingcheng 125100, China; ${ }^{3}$ Department of Gastroenterology, General Hospital of Shenyang Military Area, Shenyang 110000, China; \\ ${ }^{4}$ Depto. de Clínica Médica - Faculdade de Medicina de Botucatu/UNESP Av. Prof. Mário Rubens Guimarães Montenegro, s/n Distrito de Rubião \\ Jr. s/n Botucatu - SP CEP 18618687, Brazil; ${ }^{5}$ Medicina Interna 1, Azienda di Rilievo Nazionale ad Alta Specializzazione Civico - Di Cristina - \\ Benfratelli, Piazzale Leotta 4, 90100, Palermo, Italy; ${ }^{6}$ Epatologia e Gastroenterologia, Ospedale Niguarda Ca' Granda, Piazza Ospedale Maggiore 3 , \\ 20162 Milano, Italy \\ Contributions: (I) Conception and design: X Qi, L Shao; (II) Administrative support: X Qi, L Shao; (III) Provision of study materials or patients: X Qi, \\ L Shao; (IV) Collection and assembly of data: B Han, J Ma; (V) Data analysis and interpretation: B Han, S An; (VI) Manuscript writing: All authors; \\ (VII) Final approval of manuscript: All authors. \\ *These authors contributed equally to this work. \\ Correspondence to: Prof. Lichun Shao. Department of Gastroenterology, No. 463 Hospital of Chinese PLA, No. 46 Xiaoheyan Road, Shenyang \\ 110000, China. Email: slc700214@163.com; Dr. Xingshun Qi. Department of Gastroenterology, General Hospital of Shenyang Military Area, \\ No. 83 Wenhua Road, Shenyang 110000, China. Email: xingshunqi@126.com.
}

Background: To evaluate the ability of albumin-to-bilirubin (ALBI) score for assessing the in-hospital death in cirrhotic patients.

Methods: Overall, 1,067 cirrhotic patients admitted between January 2009 and December 2014 were retrospectively enrolled. We calculated the Child-Pugh, model for end-stage liver disease (MELD), and ALBI scores. We performed receiver operating characteristic curve (ROC) analyses to assess the in-hospital death. We calculated the area under the ROC curve (AUC).

Results: In the overall analysis, all of the three scores can significantly assess the in-hospital death (ChildPugh score AUC =0.750, 95\% CI: 0.713-0.784, P<0.0001; MELD score AUC =0.728, 95\% CI: 0.689-0.765, $\mathrm{P}<0.0001$; ALBI score AUC $=0.698,95 \% \mathrm{CI}: 0.667-0.727, \mathrm{P}<0.0001)$. In the subgroup analysis of hepatitis $\mathrm{B}$ virus, Child-Pugh and ALBI scores were suitable to assess in-hospital death (Child-Pugh score AUC =0.752, 95\% CI: 0.679-0.816, $\mathrm{P}<0.0001$; ALBI score AUC $=0.803$, 95\% CI: 0.751-0.849, $\mathrm{P}=0.0002$ ) and both were superior to the MELD score (AUC=0.564, 95\% CI: 0.483-0.643, P=0.5357). In the subgroup analysis of alcohol abuse, Child-Pugh and MELD scores properly assessed in-hospital death (Child-Pugh score AUC $=0.791,95 \%$ CI: 0.727-0.846, P<0.0001; MELD score AUC =0.720, 95\% CI: 0.647-0.786, P=0.0023), rather than ALBI score (AUC =0.646, 95\% CI: 0.588-0.702, $\mathrm{P}=0.1360$ ).

Conclusions: ALBI score might be an alternative index for assessing the in-hospital death in patients with liver cirrhosis.

Keywords: Child-Pugh; model for end-stage liver disease (MELD); albumin-to-bilirubin (ALBI); liver cirrhosis; prognosis

Received: 21 August 2017; Accepted: 07 September 2017; Published: 07 November 2017.

doi: $10.21037 / \operatorname{tgh} .2017 .09 .11$

View this article at: http://dx.doi.org/10.21037/tgh.2017.09.11 


\section{Introduction}

Liver cirrhosis is a common cause of death in the world $(1,2)$. The accurate prognostic assessment of liver cirrhosis is important in our clinical practice. Child-Pugh score is the most commonly used tool to predict the prognosis of liver cirrhosis (3). However, it has been established for a long time, and its components are selected primarily based on the surgeons' experiences. Model for end-stage liver disease (MELD) score is also a central tool for prognostic assessment of liver cirrhosis $(4,5)$. It has greatly changed the landscape of liver transplantation donor allocation (6), and its components are determined according to the strict statistical analyses. Until now, there are lots of controversy regarding the comparison of Child-Pugh versus MELD scores (7-10). More recently, albumin-to-bilirubin (ALBI) score has been proposed as a novel, simple, and readily available model for assessing the severity of liver dysfunction in hepatocellular carcinoma (11). Several studies have also explored the prognostic role of ALBI score in other liver diseases (12-15). Herein, we attempted to conduct a large study to assess the ALBI score for in-hospital death in unselected cirrhotic patients.

\section{Methods}

\section{Patients}

The study protocol of this retrospective study has been approved by the ethical committee of the No. 463 hospital of Chinese PLA, which is a tertiary hospital. In this study, the researchers enrolled all cirrhotic patients admitted to the No. 463 hospital of Chinese PLA from January 2009 to December 2014. Two researchers (J Ma and B Han) collected the primary data, including age, sex, cirrhosis etiology, major causes of admission, liver function, renal function, coagulation function, and in-hospital outcome, from electronic medical charts. One of them also validated the accuracy of data. We calculated the Child-Pugh, MELD, and ALBI scores according to the current formulas $(3,5,11)$.

\section{Data analysis}

First, the baseline characteristics of all patients, patients with hepatitis B virus infection, and patients with alcohol abuse were summarized. As for the baseline data, we calculated the continuous data as the mean \pm standard deviation and median (range) and the categorical data as the frequency (percentage). Second, we evaluate the suitability of the different scores for assessing the in-hospital death according to the receiver operating characteristic curve (ROC) analysis. We calculated the areas under the ROC curve (AUC) with 95\% confidence intervals (CI). The cutoff values were defined in line with the best sensitivity and specificity. The corresponding sensitivity, specificity, and positive and negative likelihood ratios were also reported. Then, the diagnostic accuracy of the three different scores were compared by the De-Long test. We used the MedCalc statistical software to undergo the data analyses.

\section{Results}

\section{Overall analysis}

We included 1,067 cirrhotic patients. Baseline characteristics were summarized in Table 1 . Briefly, $67.9 \%$ of patients were male; $29 \%$ and $30.9 \%$ of patients had hepatitis B virus infection and history of alcohol abuse, respectively. The most common cause of admission was ascites (65.4\%).

Child-Pugh score could be evaluated in 591 patients. Most of them were graded as Child B (49.6\%) or C (40.4\%). Twentysix out of the 591 patients died during their hospitalizations (4.39\%). The AUC was 0.750 (95\% CI: 0.713-0.784, $\mathrm{P}<0.0001$ ) (Figure 1A). The best cut-off value was 9 with a sensitivity of 80.77 (95\% CI: 60.6-93.4), a specificity of 64.07 (95\% CI: $60.0-68.0$ ), a positive likelihood ratio of 2.25 , and a negative likelihood ratio of 0.30 .

MELD score could be evaluated in 549 patients. Median MELD score was 10.96 (range: -1.89-38.86). Twenty-six out of the 549 patients died during their hospitalizations (4.73\%). The AUC was 0.728 (95\% CI: 0.689-0.765, $\mathrm{P}<0.0001$ ) (Figure $1 B$ ). The best cut-off value was 12.9787 with a sensitivity of 84.62 (95\% CI: 65.1-95.6), a specificity of 62.72 (95\% CI: 58.4-66.9), a positive likelihood ratio of 2.27, and a negative likelihood ratio of 0.25 .

ALBI score could be evaluated in 926 patients. Median ALBI score was -0.190 (range: $-2.320-1.370$ ). Thirty-two out of the 926 patients died during their hospitalizations (3.45\%). The AUC was 0.698 (95\% CI: 0.667-0.727, $\mathrm{P}<0.0001$ ) (Figure $1 C$ ). The best cut-off value was 0.1 with a sensitivity of 71.87 (95\% CI: $53.3-86.3$ ), a specificity of 66.22 (95\% CI: 63.0-69.3), a positive likelihood ratio of 2.13 , and a negative likelihood ratio of 0.42 .

ALBI, Child-Pugh, and MELD scores could be evaluated in 548 patients. Twenty-six of the 548 patients died during their hospitalizations. The largest AUC (0.746) was 
Table 1 Baseline characteristics

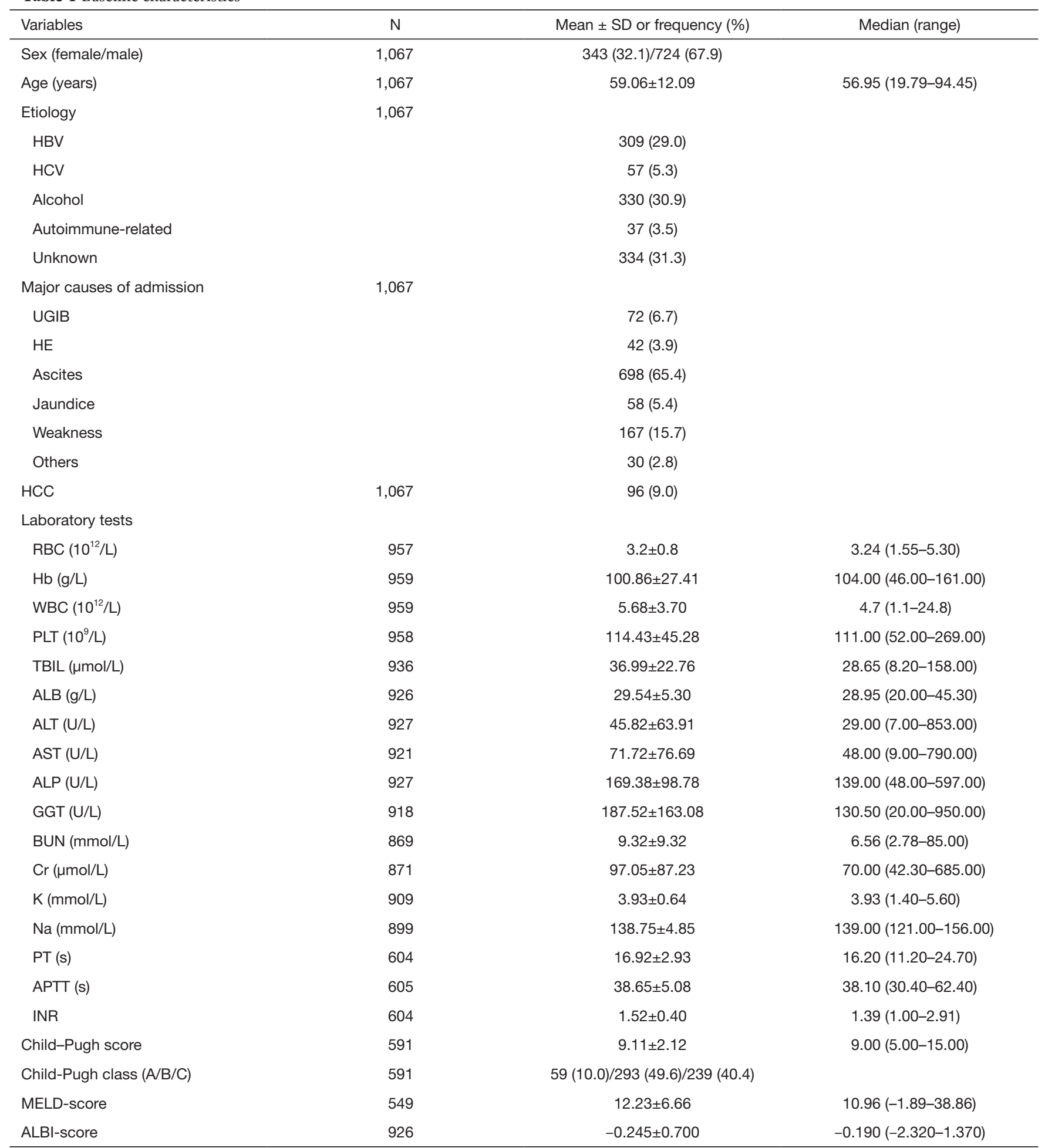

HBV, hepatitis B virus; HCV, hepatitis C virus; UGIB, upper gastrointestinal bleeding; HE, hepatic encephalopathy; HCC, hepatocellular carcinoma; RBC, red blood cell; Hb, hemoglobin; WBC, white blood cell; PLT, platelet; TBIL, total bilirubin; ALT, alanine aminotransferase; ALB, albumin; ALP, alkaline phosphatase; AST, aspartate aminotransferase; BUN, blood urea nitrogen; Cr, creatinine; K, potassium ion; $\mathrm{Na}$, sodium ion; GGT, gamma-glutamyl transpeptidase; INR, international normalized ratio; PT, prothrombin time; APTT, activated partial thromboplastin time; MELD, model for end stage liver disease; ALBI, albumin-bilirubin. 

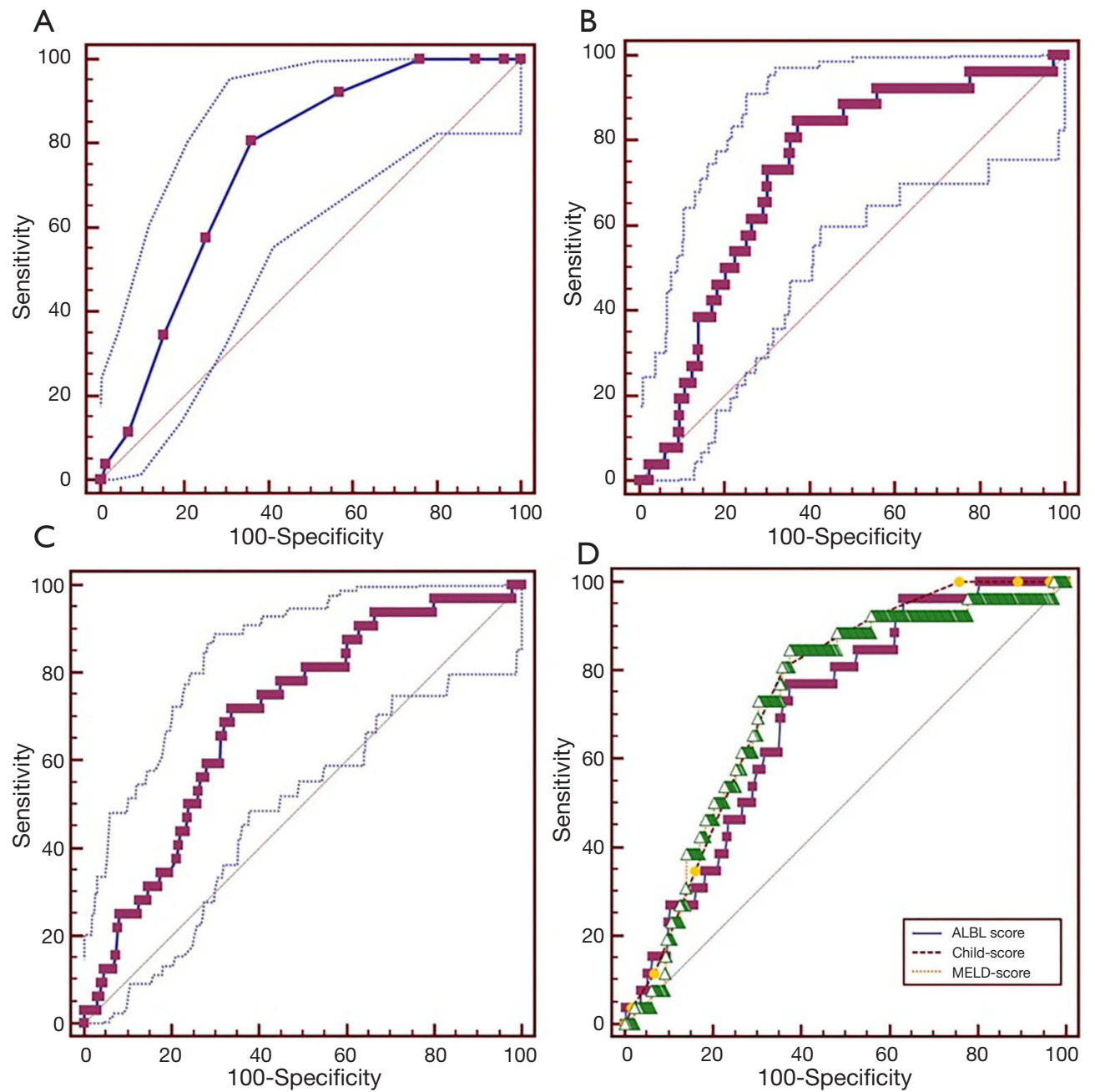

Figure 1 ROC analyses of Child-Pugh (A), MELD (B), and ALBI (C) scores for predicting the in-hospital mortality of liver cirrhosis. A comparison of their prognostic abilities is also presented (D). ROC, receiver operating characteristic curve; MELD, model for end stage liver disease; ALBI, albumin-bilirubin.

obtained by Child-Pugh score, followed by MELD (0.728) and ALBI scores (0.703). The difference between them was not statistically significant (ALBI vs. Child-Pugh, $\mathrm{P}=0.2400$; ALBI vs. MELD, $\mathrm{P}=0.6463$; Figure 1D).

\section{Subgroup analysis of hepatitis B patients}

We included 309 cirrhotic patients with hepatitis B virus. Baseline characteristics of these patients were summarized in Table 2. Briefly, a majority of patients were male (74.1\%). The most common cause of admission was ascites (63.4\%).

Child-Pugh score could be evaluated in 167 patients. Most of them were graded as Child B (44.3\%) or C (43.1\%). Six out of the 167 patients died during their hospitalizations
(3.59\%). The AUC was 0.752 (95\% CI: 0.679-0.816, $\mathrm{P}<0.0001$ ) (Figure $2 A$ ). The best cut-off value was 9 with a sensitivity of 100 (95\% CI: 54.1-100), a specificity of 60.87 (95\% CI: 52.9-68.5), a positive likelihood ratio of 2.56, and a negative likelihood ratio of 0.00 .

MELD score could be evaluated in 159 patients. Median MELD score was 12.85 (range: 1.23-38.86). Six out of the 159 patients died during their hospitalizations (3.77\%). The AUC of MELD score was 0.564 (95\% CI: 0.483-0.643, $\mathrm{P}=0.5357$ ) (Figure $2 B$ ). The best cut-off value of MELD score was 10.7139 with a sensitivity of 83.33 (95\% CI: 35.9-99.6), a specificity of 43.79 (95\% CI: 35.8-52.0), a positive likelihood ratio of 1.48 , and a negative likelihood ratio of 0.38 .

ALBI score could be evaluated in 267 patients. Median 
Table 2 Baseline characteristics in HBV group

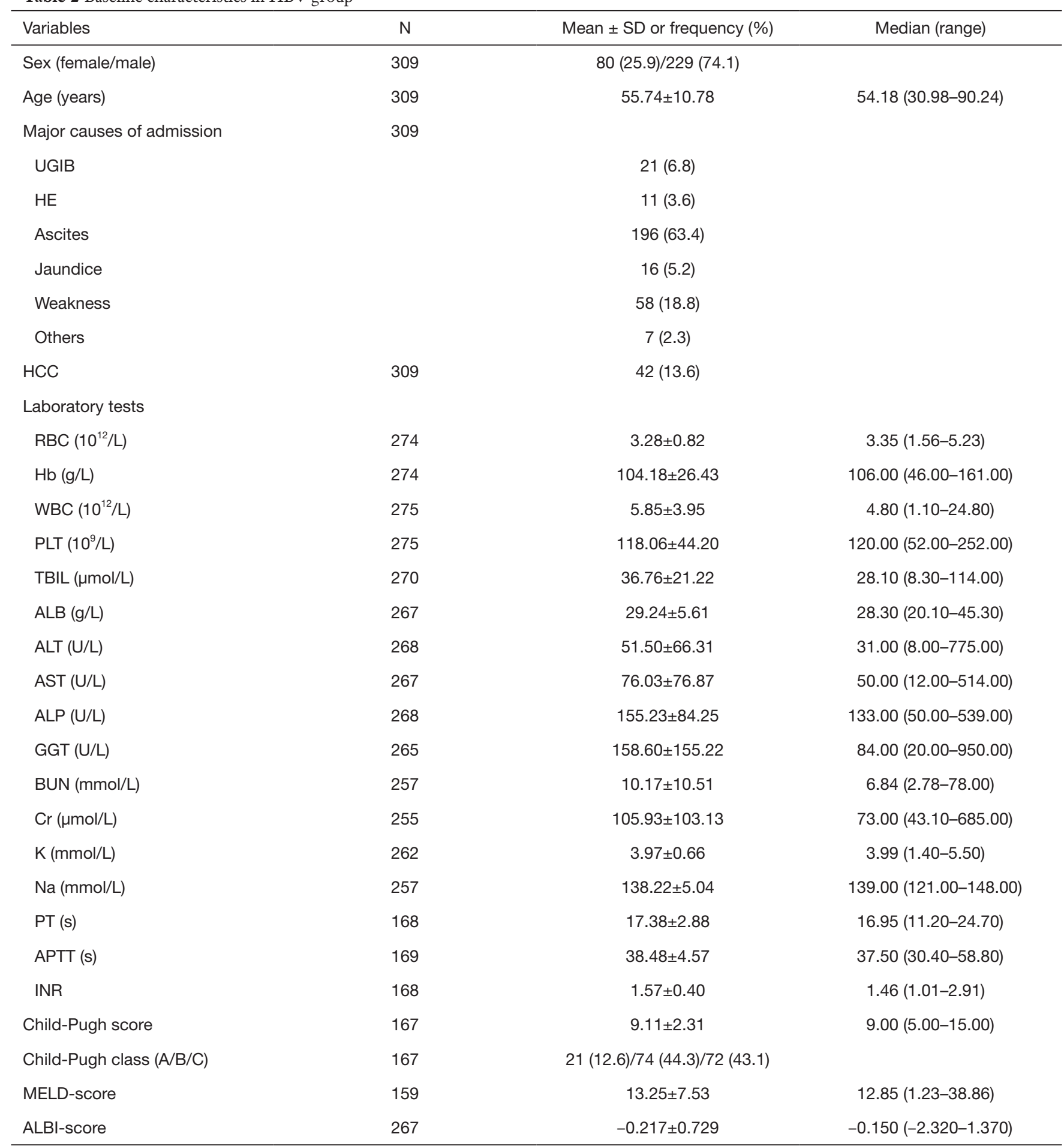

HBV, hepatitis B virus; UGIB, upper gastrointestinal bleeding; HE, hepatic encephalopathy; HCC, hepatocellular carcinoma; RBC, red blood cell; Hb, hemoglobin; WBC, white blood cell; PLT, platelet; TBIL, total bilirubin; ALB, albumin; ALT, alanine aminotransferase; AST, aspartate aminotransferase; ALP, alkaline phosphatase; GGT, gamma-glutamyl transpeptidase; BUN, blood urea nitrogen; Cr, creatinine; $\mathrm{K}$, potassium ion; Na, sodium ion; PT, prothrombin time; APTT, activated partial thromboplastin time; INR, international normalized ratio; MELD, model for end stage liver disease; ALBI, albumin-bilirubin. 

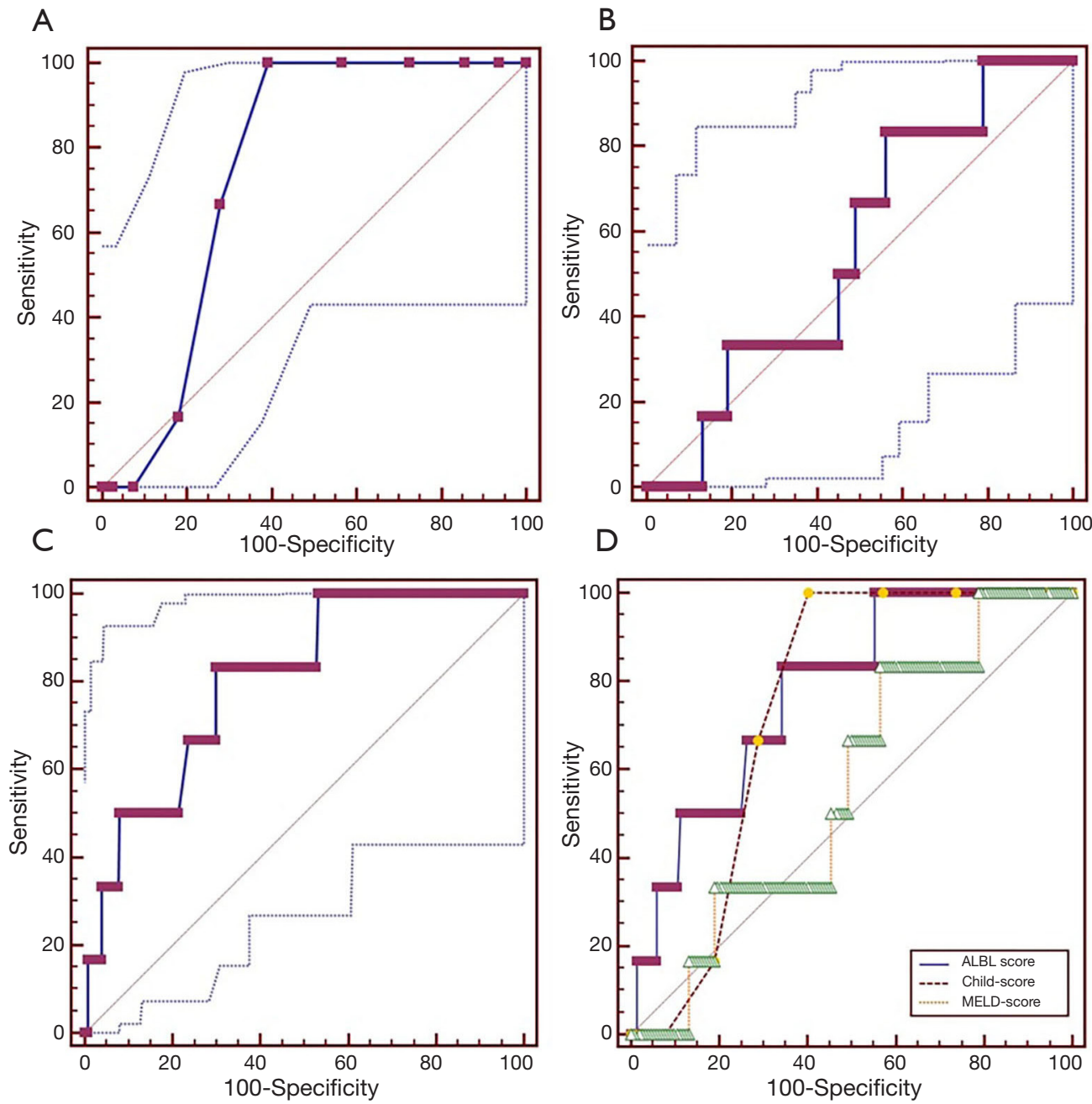

Figure 2 ROC analyses of Child-Pugh (A), MELD (B), and ALBI (C) scores for predicting the in-hospital mortality of hepatitis B virus related liver cirrhosis. A comparison of their prognostic abilities is also presented (D). ROC, receiver operating characteristic curve; MELD, model for end stage liver disease; ALBI, albumin-bilirubin.

ALBI score was -0.150 (range: $-2.320-1.370$ ). Six out of the 267 patients died during their hospitalizations (2.24\%). The AUC was 0.803 (95\% CI: $0.751-0.849, \mathrm{P}=0.0002$ ) (Figure $2 C$ ). The best cut-off value was 0.26 with a sensitivity of 83.33 (95\% CI: 35.99-99.6), a specificity of 70.11 (95\% CI: 64.2-75.6), a positive likelihood ratio of 2.79 , and a negative likelihood ratio of 0.24 .

ALBI, Child-Pugh, and MELD scores could be evaluated in 158 patients. Six out of the 158 patients died during their hospitalizations (3.79\%). The largest AUC was obtained by ALBI score (0.778), followed by Child-Pugh (0.742) and MELD scores (0.562). The AUC of ALBI score was significantly larger than that of MELD score $(\mathrm{P}=0.0007)$, but was similar to that of Child-Pugh score $(\mathrm{P}=0.6577)$ (Figure 2D).

\section{Subgroup analysis of alcobol abuse patients}

We included 330 patients with alcohol abuse. Baseline characteristics of these patients were summarized in Table 3. Briefly, nearly all patients were male (98.2\%). The most common cause of admission was ascites (69.1\%).

Child-Pugh score could be evaluated in 191 patients. Most of them were graded as Child B (47.6\%) or C $(44.0 \%)$. Seven out of the 191 patients died during their hospitalizations (3.66\%). The AUC was 0.791 (95\% CI: 
Table 3 Baseline characteristics in alcohol group

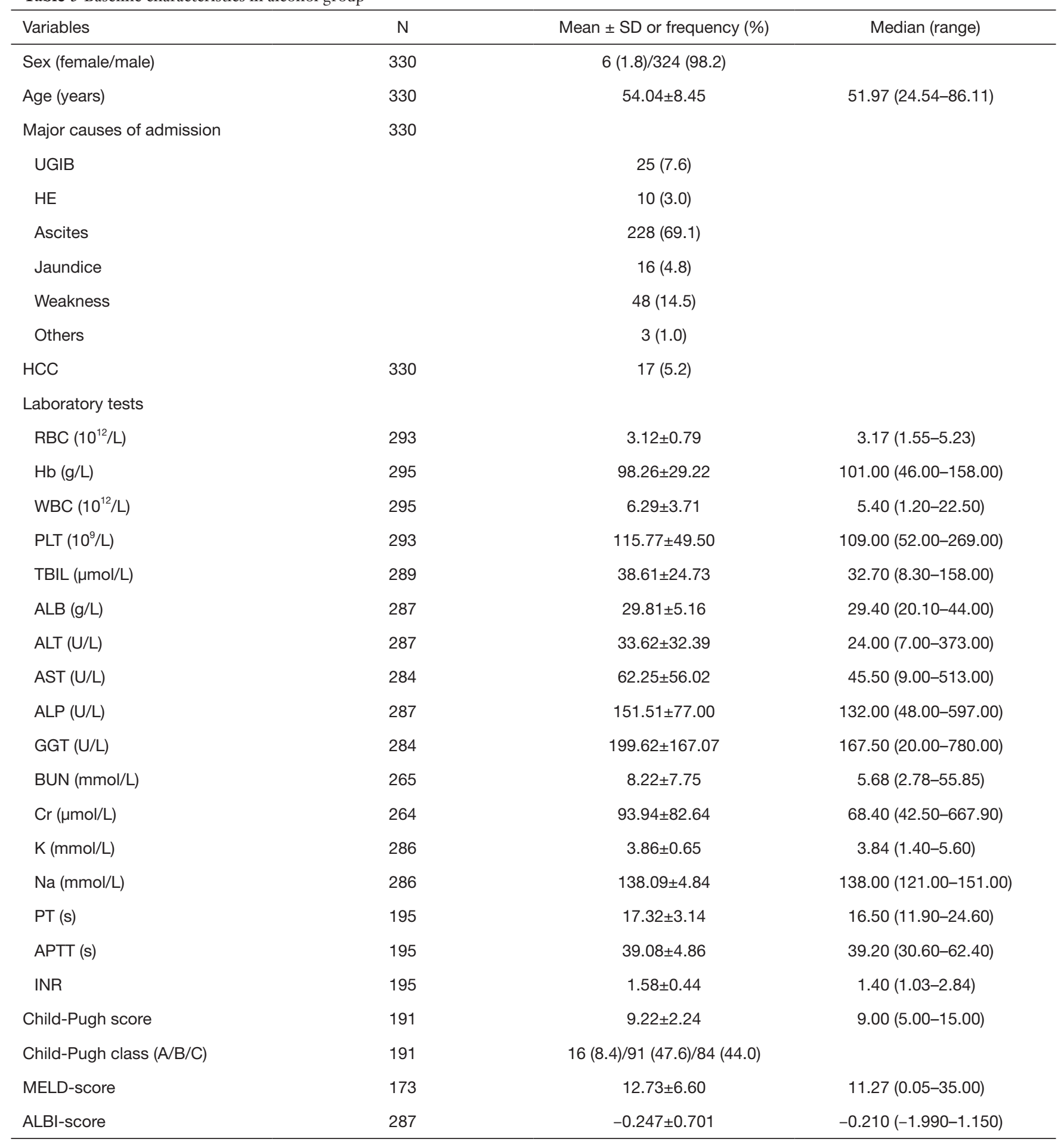

UGIB, upper gastrointestinal bleeding; HE, hepatic encephalopathy; HCC, hepatocellular carcinoma; RBC, red blood cell; Hb, hemoglobin; WBC, white blood cell; PLT, platelet; TBIL, total bilirubin; ALB, albumin; ALT, alanine aminotransferase; AST, aspartate aminotransferase; ALP, alkaline phosphatase; GGT, gamma-glutamyl transpeptidase; BUN, blood urea nitrogen; Cr, creatinine; K, potassium ion; Na, sodium ion; PT, prothrombin time; APTT, activated partial thromboplastin time; INR, international normalized ratio; MELD, model for end stage liver disease; ALBI, albumin-bilirubin. 
A
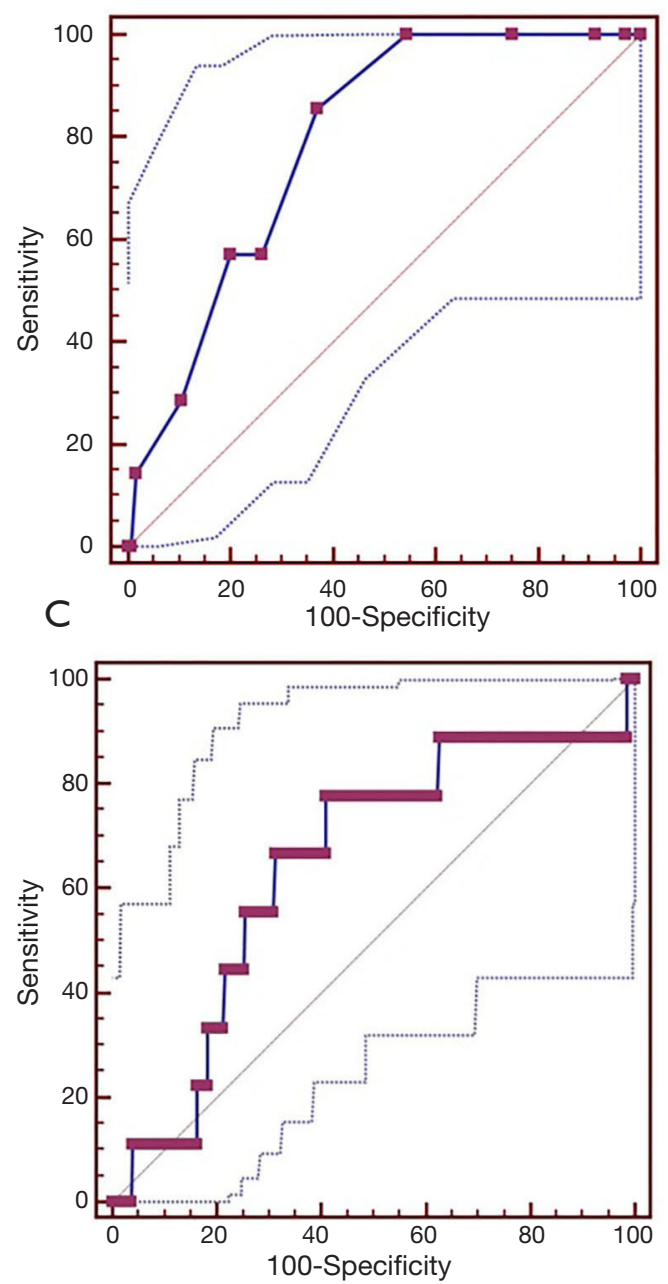

B
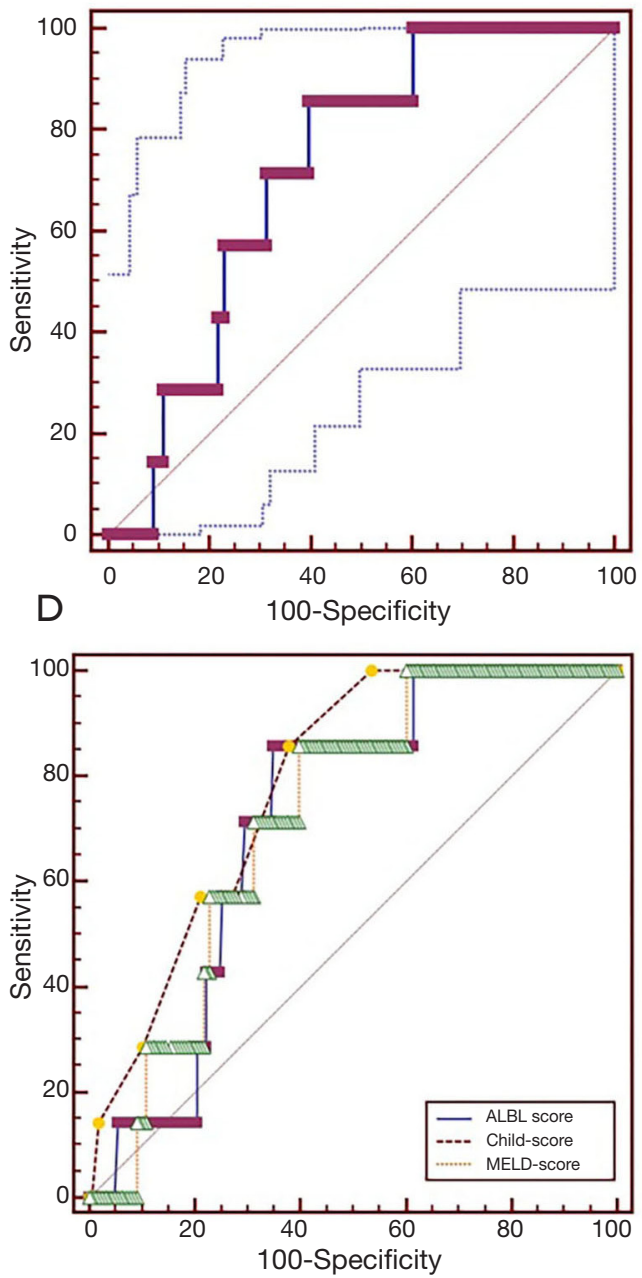

Figure 3 ROC analyses of Child-Pugh (A), MELD (B), and ALBI (C) scores for predicting the in-hospital mortality of alcohol related liver cirrhosis. A comparison of their prognostic abilities is also presented (D). ROC, receiver operating characteristic curve; MELD, model for end stage liver disease; ALBI, albumin-bilirubin.

$0.727-0.846, \mathrm{P}<0.0001$ ) (Figure $3 A$ ). The best cut-off value was 9 with a sensitivity of 85.71 (95\% CI: 42.1-99.6), a specificity of 63.04 (95\% CI: 55.6-70.0), a positive likelihood ratio of 2.32, and a negative likelihood ratio of 0.23 .

MELD score could be evaluated in 173 patients. Median MELD score was 11.27 (range: 0.05-35). Seven out of the 173 patients died during their hospitalizations (4.04\%). The AUC was 0.720 (95\% CI: 0.647-0.786, $\mathrm{P}=0.0023$ ) (Figure 3B). The best cut-off value was 13.1305 with a sensitivity of 85.71 (95\% CI: 42.1-99.6), a specificity of 60.24 (95\% CI: 52.4-67.7), a positive likelihood ratio of 2.16, and a negative likelihood ratio of 0.24 .

ALBI score could be evaluated in 287 patients. Median ALBI score was -0.210 (range: $-1.990-1.150$ ). Nine out of the 287 patients died during their hospitalizations (3.13\%). The AUC was 0.646 (95\% CI: 0.588-0.702, $\mathrm{P}=0.1360$ ) (Figure 3C). The best cut-off value was -0.04 with a sensitivity of 77.78 (95\% CI: 40.0-97.2), a specificity of 58.99 (95\% CI: 53.0-64.8), a positive likelihood ratio of 1.90, and a negative likelihood ratio of 0.38 .

ALBI, Child-Pugh, and MELD scores could be evaluated in 173 patients. Seven out of the 173 patients died during their hospitalizations (4.04\%). The largest AUC (0.787) was obtained by Child-Pugh score, followed by MELD (0.720) and ALBI scores (0.717). The difference between them was not statistically significant (ALBI vs. Child-Pugh, $\mathrm{P}=0.3037$; ALBI vs. MELD, $\mathrm{P}=0.9607$; Figure 3D). 


\section{Discussion}

In a recent systematic review and meta-analysis by Peng et al., 119 papers, which compared the discriminative ability of Child-Pugh versus MELD scores, were identified (16). They performed the comparison of Child-Pugh versus MELD scores according to the patient status, clinical presentations, and treatment options. They suggested similar prognostic abilities of Child-Pugh and MELD scores in most of cases. However, their benefits and drawbacks in specific conditions should be further identified. In the present study, a large number of cirrhotic patients were selected. Most of our patients had severe liver dysfunction. The overall analysis confirmed that ChildPugh and MELD scores were very similar for assessing the in-hospital death in cirrhosis, both of them achieving moderate prognostic abilities. The subgroup analyses of hepatitis B patients showed that Child-Pugh score might be better than MELD score for predicting the in-hospital death, but the difference between them was not statistically significant. Furthermore, the prognostic abilities of ChildPugh and MELD scores remained similar in patients with alcohol related liver cirrhosis. Notably, in patients with hepatitis B, the prognostic ability of MELD score was not significant. Additionally, considering the potential superiority of Child-Pugh score over MELD score in such patients, three components (i.e., ascites, albumin and hepatic encephalopathy), which are not included in the MELD score, should be of prognostic importance.

ALBI score may be an alternative for assessing the grade of liver dysfunction. Our study suggests that ALBI score may be the most readily available prognostic model, followed by Child-Pugh score and MELD score. This is primarily because only two convenient parameters are necessary for the ALBI score. The overall analysis also demonstrated that the ability of ALBI score was similar to that of Child-Pugh and MELD score for assessing the inhospital death. Notably, the subgroup analysis of hepatitis $B$ patients showed that the prognostic role of ALBI score was significantly superior to that of MELD score. These findings indicated that ALBI score should be widely spread in our clinical practice.

Our study had several limitations. The major one is the potential bias of patient selection. First, not all medical charts had enough data for calculating Child-Pugh, MELD, and ALBI scores. Indeed, about half of patients had the data regarding Child-Pugh and MELD scores. In the overall analysis, only $55 \%(591 / 1,067)$ of patients had data regarding Child-Pugh score, and only 51\% (549/1,067) of patients had data regarding MELD score. In the subgroup analysis of patients with hepatitis B, 54\% (167/309) of patients had data regarding Child-Pugh score, and only $51 \%(159 / 309)$ of patients had data regarding MELD score. In the subgroup analysis of patients with alcohol related liver cirrhosis, $58 \%(191 / 330)$ of patients had data regarding Child-Pugh score, and only 52\% (173/330) of patients had data regarding MELD score. Second, the data were retrospectively collected. Third, the major cause of admission was heterogeneous among patients.

In conclusion, ALBI score may be a reliable alternative prognostic model for assessing liver cirrhosis severity, especially in patients with hepatitis B.

\section{Acknowledgements}

None.

\section{Footnote}

Conflicts of Interest: The authors have no conflicts of interest to declare.

Ethical Statement: The study protocol of this retrospective study has been approved by the ethical committee of the No. 463 hospital of Chinese PLA [NO. K(2016)001].

\section{References}

1. Blachier M, Leleu H, Peck-Radosavljevic M, et al. The burden of liver disease in Europe: a review of available epidemiological data. J Hepatol 2013;58:593-608.

2. Lozano R, Naghavi M, Foreman K, et al. Global and regional mortality from 235 causes of death for 20 age groups in 1990 and 2010: a systematic analysis for the Global Burden of Disease Study 2010. Lancet 2012;380:2095-128.

3. Pugh RN, Murray-Lyon IM, Dawson JL, et al. Transection of the oesophagus for bleeding oesophageal varices. Br J Surg 1973;60:646-9.

4. Kamath PS, Wiesner RH, Malinchoc M, et al. A model to predict survival in patients with end-stage liver disease. Hepatology 2001;33:464-70.

5. Kamath PS, Kim WR. The model for end-stage liver disease (MELD). Hepatology 2007;45:797-805.

6. Gambato M, Senzolo M, Canova D, et al. Algorithm for prioritization of patients on the waiting list for liver 
transplantation. Transplant Proc 2007;39:1855-6.

7. Gheorghe L, Iacob S, Simionov I, et al. Natural history of compensated viral B and D cirrhosis. Rom J Gastroenterol 2005;14:329-35.

8. Cholongitas E, Marelli L, Shusang V, et al. A systematic review of the performance of the model for end-stage liver disease (MELD) in the setting of liver transplantation. Liver Transpl 2006;12:1049-61.

9. Peng Y, Qi X, Dai J, et al. Child-Pugh versus MELD score for predicting the in-hospital mortality of acute upper gastrointestinal bleeding in liver cirrhosis. Int J Clin Exp Med 2015;8:751-7.

10. Chalasani N, Kahi C, Francois F, et al. Model for endstage liver disease (MELD) for predicting mortality in patients with acute variceal bleeding. Hepatology 2002;35:1282-4.

11. Johnson PJ, Berhane S, Kagebayashi C, et al. Assessment of liver function in patients with hepatocellular carcinoma: a new evidence-based approach-the ALBI grade. J Clin

doi: 10.21037/tgh.2017.09.11

Cite this article as: Shao L, Han B, An S, Ma J, Guo X, Romeiro FG, Mancuso A, Qi X. Albumin-to-bilirubin score for assessing the in-hospital death in cirrhosis. Transl Gastroenterol Hepatol 2017;2:88.
Oncol 2015;33:550-8.

12. Chan AW, Chan RC, Wong GL, et al. New simple prognostic score for primary biliary cirrhosis: Albuminbilirubin score. J Gastroenterol Hepatol 2015;30:1391-6.

13. Chen RC, Cai YJ, Wu JM, et al. Usefulness of albuminbilirubin grade for evaluation of long-term prognosis for hepatitis B-related cirrhosis. J Viral Hepat 2017;24:238-45.

14. Zou D, Qi X, Zhu C, et al. Albumin-bilirubin score for predicting the in-hospital mortality of acute upper gastrointestinal bleeding in liver cirrhosis: A retrospective study. Turk J Gastroenterol 2016;27:180-6.

15. Peng Y, Qi X, Tang S, et al. Child-Pugh, MELD, and ALBI scores for predicting the in-hospital mortality in cirrhotic patients with acute-on-chronic liver failure. Expert Rev Gastroenterol Hepatol 2016;10:971-80.

16. Peng Y, Qi X, Guo X. Child-Pugh Versus MELD Score for the Assessment of Prognosis in Liver Cirrhosis: A Systematic Review and Meta-Analysis of Observational Studies. Medicine (Baltimore) 2016;95:e2877. 